\title{
The challenges of physician retirement
}

\author{
Cite as: CMAJ 2017 January 16;189:E90-1. doi: 10.1503/cmaj.109-5356
}



reparing for retirement poses challenges for many working adults, but for physicians, the concerns go beyond finances and planning how to fill their days.

"The main challenge confronting physicians who are seeking to retire is the justified concern for the future wellbeing of their patients," Dr. Alan Ruddiman, president of Doctors of BC, said in an email.

Physicians most often expect to retire around age 60 , but actually retire closer to age 69 , according to a systematic review of 65 studies published on Nov. 15 in Human Resources for Health. Most of the studies examined were conducted in the United States, the United Kingdom, Canada and Australia between 1978 and 2015. In many countries, a pattern has emerged over the past 40 years whereby a "disproportionate" number of physicians practise medicine beyond the traditional retirement age of 65 .

Retirement planning and transition strategies have "important implications for patients, hospitals and health care systems," states the report. When physicians retire earlier than expected or, on the other end of the spectrum, hang onto their stethoscopes a little too long, it can have "dire consequences in terms of both patient safety and human resources allocations."

Reasons commonly cited for early retirement include burnout, a lost sense of autonomy and health concerns, such as psychological stress. Physicians who delay retirement reported doing so because of concern for their patients, a lack of interests outside medicine, financial obligations and a fear of losing their sense of identity, among other reasons.

According to the report, physician retirement tends to be a difficult subject for hospitals and other medical facilities to broach. They must strike a "delicate balance between encouraging prepara-

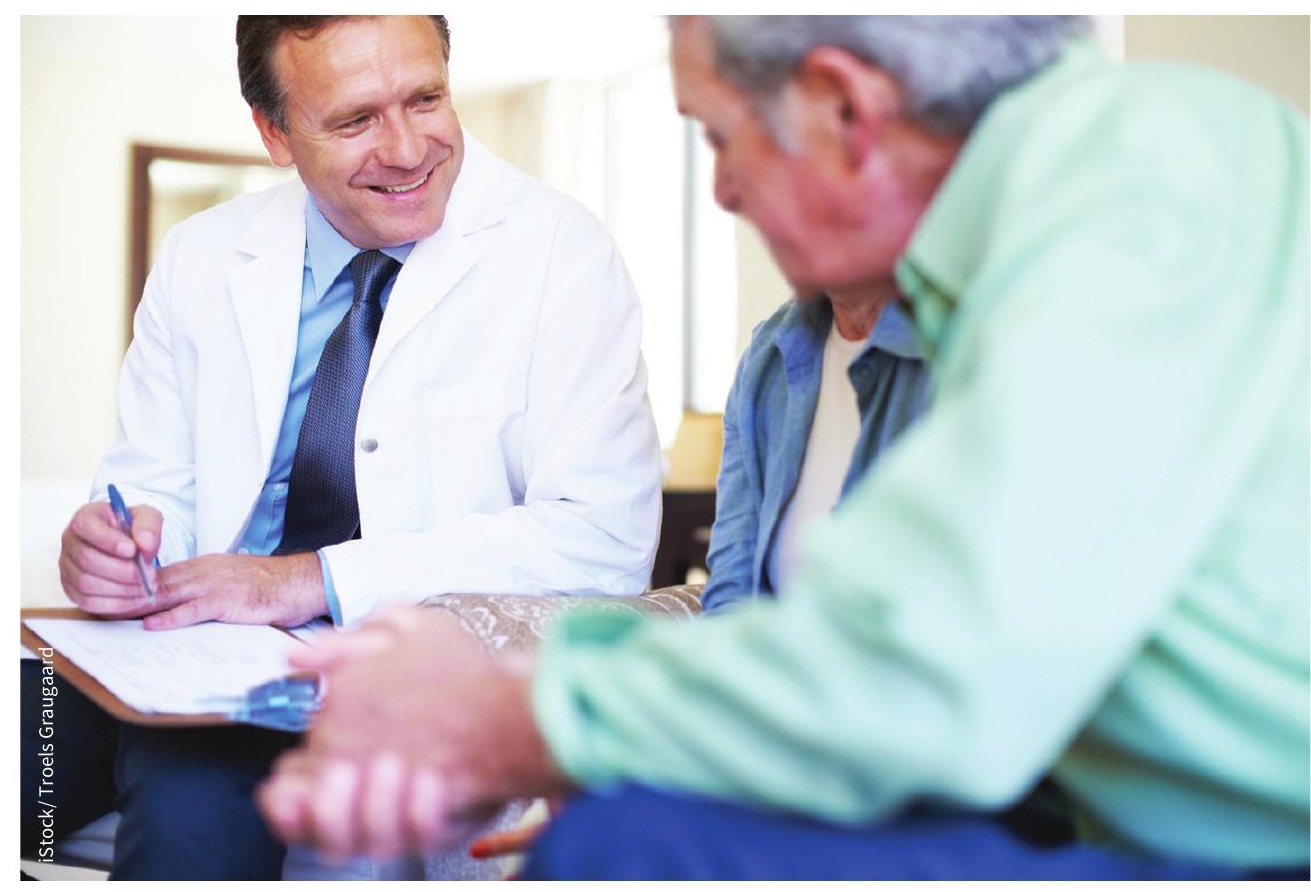

The end of long-term relationships between family physicians and patients upon a doctor's retirement can lead to patient anxiety.

tion for retirement and delaying the timing and eventual transitions of its most experienced staff who will be replaced by a growing pool of younger physicians who stand waiting in the wings for professional opportunities."

\section{Help for family physicians}

To assist family physicians in this transition, Doctors of $B C$ recently posted an article on retirement on its website. It also pointed physicians to the "How to Retire Guide" by the Vancouver Division of Family Practice, a nonprofit "led by and for Vancouver-area family doctors" with more than 900 members. The guide offers practical advice on several areas of physician retirement, including financial planning, transitioning patients to new physicians, preparing a medical practice for takeover by another doctor and providing adequate notice to patients before closing a practice.
Ideally, every retiring family physician would have a suitable peer already lined up to take over their workload. But this is much easier said than done. For a doctor with a large practice, it can prove particualready in short supply in many areas.

Another issue is the demographics of the family practice. Many patients of older doctors are older themselves, having aged along side their physicians, and have all the health complications often associated with old age. Taking on all these patients may be perceived as daunting for younger physicians. As for the patients, the idea of switching to a new doctor after decades at the same practice can lead to considerable stress.

"These relationships are founded on trust and the privacy of the doctorpatient relationship," said Ruddiman. "The very thought of needing to start over with a new doctor can be quite perlarly challenging. Family physicians are 
plexing to patients, or worse yet, the uncertainty and possibility that they won't be able to find a new doctor can cause patients great anxiety."

The patients may not be the only anxious ones, according to Dr. Terrie Brandon, clinical director of the physician and family support program for the Alberta Medical Association. "Many physicians also struggle with the emotional aspect of retirement as their sense of self is strongly associated with the work they do and they have a strong emotional bond with their patients," Brandon said in an email. "Many fear that retirement will not provide the intellectual stimulation that they enjoy in their work."

Indeed, family physicians are well known for their dedication to their profession and to patients. That is why many doc- tors transition slowly into retirement, rather than going from 60-plus hours of work a week to zero overnight.

"Practising medicine is often an identity as much as it is a job or career. Combine that with other retirement changes and physicians often ease into it by continuing to work part-time in some aspect of the medical field," Lowell Thiessen, wealth lead-financial services for MD Financial Management, said in an email. "It is worth thinking through not only what you are retiring from, but what you are retiring to. Beyond compensation, what provides meaning to you as a practising physician and how will you replace that in retirement?"

As for the financial aspects of retirement, physicians have some unique challenges in that department as well. First of all, saving for retirement early in their careers can be difficult because of the large student debts most physicians carry out of medical school and residency. Yet personal savings for retirement are more important in medicine than in many other professions, because most doctors are selfemployed and will have fewer sources of secure retirement income, such as company pensions.

"Winding up a business, in addition to dealing with other retirement challenges, whether professional or personal, can be emotional and stressful," said Thiessen. "We advise that physicians work with their financial advisor, tax specialist and lawyer to help make the best decisions for their business, employees and patients."

Roger Collier, CMAJ 